\title{
Molecular Modeling of the Elastomeric Properties of Repeating Units and Building Blocks of Resilin, a Disordered Elastic Protein+
}

\author{
Md Shahriar K Khandaker ${ }^{a}$, Daniel M. Dudek, Eric P. Beers ${ }^{b}$, David A. Dillard ${ }^{c}$ and David R. Bevan* ${ }^{d}$
}

\footnotetext{
a. Department of Biomedical Engineering and Mechanics, Virginia Polytechnic Institute and State University, VA, USA; Email: mkkhanda@vt.edu

${ }^{b .}$ Horticulture Department; Virginia Polytechnic Institute and State University; Email: ebeers@vt.edu

c. Department of Biomedical Engineering \& Mechanics; Virginia Polytechnic Institute and State University; Email: dillard@vt.edu

d. Biochemistry Department; Virginia Polytechnic Institute and State University; E-mail: drbevan@vt.edu; Tel: (+540) 231-5040.

*Corresponding Author

tElectronic Supplementary Information (ESI) available: [Fig. S1, S2 and S3].
} 


\section{Abstract}

The mechanisms responsible for the properties of disordered elastomeric proteins are not well known. To better understand the relationship between elastomeric behavior and amino acid sequence, we investigated resilin, a disordered rubber-like protein, found in specialized regions of the cuticle of insects. Resilin of Drosophila melanogaster contains Gly-rich repetitive motifs comprised of the amino acids, PSSSYGAPGGGNGGR, which confer elastic properties to resilin. The repetitive motifs of insect resilin can be divided into smaller partially conserved building blocks: PSS, SYGAP, GGGN and GGR. Using molecular dynamics (MD) simulations, we studied the relative roles of SYGAP, and its less common variants SYSAP and TYGAP, on the elastomeric properties of resilin. Results showed that SYGAP adopts a bent structure that is one-half to one-third the end-toend length of the other motifs having an equal number of amino acids but containing SYSAP or TYGAP substituted for SYGAP. The bent structure of SYGAP forms due to conformational freedom of glycine, and hydrogen bonding within the motif apparently plays a role in maintaining this conformation. These structural features of SYGAP result in higher extensibility compared to other motifs, which may contribute to elastic properties at the macroscopic level. Overall, the results are consistent with a role for the SYGAP building block in the elastomeric properties of these disordered proteins. What we learned from simulating the repetitive motifs of resilin may be applicable to the biology and mechanics of other elastomeric biomaterials, and may provide us the deeper understanding of their unique properties.

\section{Keywords}

Resilin; Repetitive motifs; Hydrogen bonds; Molecular modeling; Disordered protein; Elastomeric proteins

\subsection{Introduction}

Elastomeric proteins are present in a wide range of living organisms, and their critical features include structural disorder and associated hydration ${ }^{1}$. Moreover, about $40 \%$ of highly structured proteins contain disordered domains ${ }^{2}$. Despite their ubiquity, we have little understanding of how molecular variations in unstructured proteins affect macroscopic material properties. The secondary structures of these proteins typically are in dynamic equilibrium between extended (poly-proline II(PPII) and $\beta$ strands) and folded (mainly $\beta$-turn) conformations ${ }^{3}$. Functional and structural properties of proteins are determined by chemical properties and sequential arrangement of their constituent amino acid residues. In depth comparisons between the amino acid sequences of ordered and disordered proteins are needed to improve the understanding of structural and functional properties of disordered proteins ${ }^{4}$. Probable disorder-promoting residues are aspartic acid, methionine, lysine, arginine, serine, glutamine, proline and glutamic acid. The majority of these disorder-promoting residues are polar, often charged, and commonly found on the surface of the proteins ${ }^{5}$. Disordered proteins usually show low sequence complexity and 
have significant amino-acid compositional bias. Glycine and proline often appear in the sequence of disordered proteins, and both contribute to disorder, though for opposite reasons ${ }^{6}$. Proline is conformationally restricted due to its cyclic side chain, promotes the formation of PPII structures, and reduces the ability to form hydrogen-bonded secondary structure ${ }^{1}$. On the other hand, glycine is so flexible due to the absence of a side chain that order is entropically unfavorable ${ }^{6}$.

Resilin is a disordered elastic protein in a family of functionally similar proteins that includes, among others, elastin, gluten, gliadin, abductin, and spider silks. Resilin can be stretched to $300 \%$ of its resting length and reportedly suffers neither from creep nor stress relaxation even after weeks of sustained straining, though experimental details are unclear $^{7-8}$. In 1962 , Jensen and Weis-Fogh ${ }^{9}$ explored the dynamic mechanical properties of resilin and found energy loss less than $5 \%$ even at 200 $\mathrm{Hz}$ at constant temperature $\left(25^{\circ} \mathrm{C}\right)$. Furthermore, resilin has been reported to have long fatigue life sustaining millions of cycles in nature without failure ${ }^{10}$. For example, in fruit flies (Drosophila species), resilin survives during the entire lifetime of the insects at 720,000 cycles per hour, with resilience higher than that of synthetic polybutadiene, a high resilience rubber ${ }^{11-12}$. Whether this long fatigue life is an inherent property of resilin or results from a natural in-vivo repair mechanism is not yet known.

First discovered in 1960 by Weis-Fogh ${ }^{7}$, resilin has remarkably diverse functions. In addition to its multiple locomotor functions ${ }^{7}$, resilin plays a role in feeding (salivary pump of assassin bugs ${ }^{13}$ ), sound production (cicada tymbal mechanism ${ }^{14}$ ), hind coxae of the froghopper ${ }^{15}$ and structural support at leg joints (cockroach ${ }^{16}$ ). Resilin works across a broad frequency range from as high as $13 \mathrm{kHz}$ in cicada ${ }^{17}$ and moth $^{18}$ sound production to as low as $6 \mathrm{~Hz}$ in cockroach locomotion ${ }^{19-20}$. Hence, resilin can be used as a spring over a wide range of speeds, and for enabling other rapid motions in insects, such as in jumping fleas, ${ }^{21-22,}$. Resilin can also be used to simplify the complex mechanism of wing-folding in insects ${ }^{23}$.

In addition to this functional diversity, resilin is also structurally diverse at both the macro- and microscopic levels of organization. It can be either a pure polymer, as in dragonfly tendons, or a composite of chitin and resilin, as in locust pre-alar arms, cockroach tarsal pads, and fruit fly wing hinges ${ }^{24}$. At the molecular level, resilin is believed to be highly unstructured with little or no secondary structures such as $\alpha$-helices or $\beta$-sheets ${ }^{24-25}$. The level of disorder in resilin is so high that when stretched to nearly the breaking point and slowly dried, it does not show any trace of crystallization ${ }^{26}$. Like other elastomeric proteins (gliadin, elastin, spider silks), resilin contains distinct repetitive domains in its polymeric sequence to confer elastic properties. These flexible polymer chains form a rubber network due to cross-linking of tyrosine residues forming di- and tri-tyrosine ${ }^{27}$. Unlike the other elastomeric proteins, the genes of resilin have both conserved and non-conserved domains across insect lineages, and the conserved domains usually participate in cross-linking. Thus, each species has its own form of resilin, likely matched to its function within that species ${ }^{28}$. 
Most insect resilin genes are comprised of three protein coding regions, or exons. Both exon 1 and exon 3 contain repeating elastomeric units while exon 2 is designated the chitin binding domain (ChBD) ${ }^{29}$. The ChBD allows strong interaction and binding between chitin and resilin during the process of resilin deposition and construction of the cuticle composite. Resilin contains distinct repetitive units, PSSSYGAPGGGNGGR in exon 1 and GYSGGRPGGQDLG in exon 3, that appear to confer elastomeric properties to proteins in ways similar to other elastomeric proteins like elastin, spider silk and gliadin ${ }^{30}$. Some insects, such as dragonfly, have a resilin gene with only a single exon homologous to exon 1, i.e. they produce pure resilin without ChBD.

There is little definitive information on the molecular basis for elastomeric properties of disordered proteins like resilin and elastin ${ }^{1}$. While both natural and recombinant forms of resilin and other elastomeric proteins have been studied, their conformational heterogeneity and insolubility have precluded the use of conventional structural determination methods, including solution NMR and X-ray crystallography ${ }^{31}$. Moreover, such proteins do not allow for investigation of the contributions of small repeating units to the properties of full length resilin ${ }^{32 .} \mathrm{MD}$ simulations have therefore been useful in developing an atomic-level description of the structure because simulations are not hindered by conformational disorder, although the scope of these studies has been limited to small oligopeptides ${ }^{33}$ and short time scales ${ }^{34}$.

To understand the extraordinary properties of resilin, a bottom-up approach can be employed by atomistic modeling ${ }^{25-26,35}$. Steered molecular dynamics (SMD) and moving constraints are two extensions of MD that allow for constant velocity pulling (e.g., moving constraints and constant velocity SMD) or constant force pulling (e.g., constant force SMD) ${ }^{36}$. In both constant velocity SMD and moving constraints scenarios, the center of mass of a group of atoms or each pulled atom is connected through a virtual spring $\left(k=\right.$ stiffness) to a virtual atom that moves at a constant velocity $(v=\text { velocity })^{37}$. The purpose of virtual atoms and virtual springs is to provide an analog to position-controlled atomic force microscopy (AFM) to compare with the experimental results ${ }^{38}$. Constant velocity SMD is one of the techniques that has been used to simulate the mechanical functions of proteins and protein unfolding pathways ${ }^{39}$, and to predict Young's modulus ${ }^{40}$.

The pulling velocity and the stiffness of the virtual springs influence the constant velocity pulling simulations. For instance, faster pulling rates can lead to over-estimated mechanical properties as they do not provide enough time for the protein to reorganize ${ }^{41}$. Though it is unclear whether this is a numerical artifact or associated with some rudimentary time dependence, the effect of pulling velocity on results for a molecule surrounded by water is not believed to be relevant to actual polymer viscoelasticity effects. Thus, a sufficiently reduced pulling velocity is employed in simulations to obtain limiting or equilibrium behavior ${ }^{26}$. However, there is a balance, which must be sought when choosing a pulling velocity, since a reduced 
pulling velocity necessitates a greater number of simulation time steps to reach the same level of deformation, which will be computationally expensive.

Moreover, periodic boundary conditions need to be applied to reduce computation time and keep the number of atoms constant in the system. If the box enclosing the protein in a solvent, is too large, most of the time will be spent on solvent interactions while a smaller box will cause boundary effects ${ }^{26}$.

Petrenko et al. ${ }^{26}$ used the NAMD2.6 molecular dynamics (MD) ${ }^{42}$ package for their simulations to assess the flexibility of simulated peptides. Using constant velocity SMD, constrained/unconstrained $M D\left(k=10 \mathrm{kcal} / \mathrm{mol} / \mathrm{A}^{02}, \mathrm{v}=0.1 \mathrm{~m} / \mathrm{s}\right)$, the CHARMM force field ${ }^{43}$ for peptides, and the TIP3P model ${ }^{44}$ for water, they reported a high level of disorder due to a lack of stable secondary structure and lack of stable intra-protein hydrogen bonds. Even when their simulations included $12 \%$ of tyrosine residues involved in crosslinks to form dityrosine, Petrenko et al. ${ }^{26}$ found no significant decrease in cross-linking due to peptide fluctuations which was consistent with the experimental work that peptide dynamics does not change with the level of crosslinking at $16 \%$ in AN16 peptides ${ }^{45}$.

In another study, Kappiyoor et al. ${ }^{25}$ used the GROMACS package ${ }^{46-47}$ and the OPLS AA force field ${ }^{48}$ for simulating the effect of polarity on the elastic properties of resilin found in different insects using constant velocity SMD $(k=$ not reported, $v=$ $0.1 \mathrm{~m} / \mathrm{s}$ ). To determine the effect of polarity, charges were varied to zero and one-half of the natural value, and all the polar amino acids were replaced with non-polar amino acids in three different simulations. Results showed that increased polarity led to higher extensibility and lower stiffness. Natural resilin is hydrophilic, and able to adsorb water to transfer deformation energy to improve elasticity while the non-polar, half charge and zero charge counterparts had higher elasticity for their hydrophobic side chains ${ }^{25}$.

Previous MD studies of resilin have not considered the function of the resilin repeating motifs or their building blocks on the elasto-mechanical properties. One work explored the effect of polarity on the elastomeric properties ${ }^{25}$ even though all natural repeating motifs in the resilin sequence have same charges while other work looked only at the absence of secondary structure $^{26}$. Consequently, MD simulations were conducted on the most frequent repeating motifs from exon 1 (PSSSYGAPGGGNGGR) compared to other repeating motifs in terms of elastomeric properties to understand their contribution to the mechanical properties of resilin. We used MD simulation to test the effects of the SYGAP (and divergent SYSAP and TYGAP) building block alone or in combination with other building blocks of the resilin repetitive motifs, e.g., SYGAP, PSSSYGAP, PSSSYGAPGGN, and PSSSYGAPGGGNGGR, to understand their effects on conformation and mechanical properties of resilin. During the process, we also studied different pulling velocities on these repeating units to choose a suitable velocity to avoid 
computationally expensive simulations, and to our knowledge this is the first study on the effect of the velocity on repetitive pentadecapeptide motifs of resilin.

The purpose of this work was to take advantage of the natural diversity of resilin to develop a fundamental understanding of the elastomeric disordered protein using MD simulations. The simulations applied to these diverse repetitive motifs will advance our understanding of the molecular mechanisms responsible for the remarkable behavior exhibited by insect resilin.

\subsection{Materials and Methods}

A bottom-up approach was employed to understand the mechanism of the elastomeric properties of resilin. The molecular models were constructed using the computer programs Discovery Studio Visualizer ${ }^{49}$ and Visual Molecular Dynamics (VMD) ${ }^{50}$. The MD package NAMD2. $6^{42}$ was used for all simulations. The all-atom CHARMM22 force field ${ }^{43}$ was used for peptides and the TIP3P model was used for water ${ }^{44}$. Covalently linked hydrogen atoms were constrained to fixed lengths with the SHAKE algorithm ${ }^{51}$ which allowed us to use an integration time step of $2 \mathrm{fs}$. For the van-der-Waals interactions, the switching function started at $10 \AA$ while the cutoff distance was $12 \AA$ bringing the interactions to zero at the cutoff distance. The system coordinates were saved every $0.01 \mathrm{~ns}$ for later analysis. To calculate long-range electrostatic forces the particle-mesh Ewald $(P M E)^{52}$ method was used. Depending on the periodic cell size, the grid point spacing for PME was adjusted to be approximately $1 \AA$.

Peptides were immersed in a water box with padding of at least $10 \AA$ on all sides. Simulations were conducted using a leapfrog time integration scheme with a time step of 0.002 picoseconds (ps). All systems were simulated using the following steps: (i) energy minimization, (ii) heating to $310 \mathrm{~K}$ (iii) a canonical NPT (constant Number of particles, Pressure, and Temperature) ensemble-based simulation performed with temperature controlled at $310 \mathrm{~K}$ for $5 \times 10^{6}$ time steps, (iv) an NVE (constant Number of particles, Volume, and Energy) equilibration, during which the motif was allowed to move freely for $5 \times 10^{6}$ time steps. Periodic boundary conditions were applied to keep the number of atoms constant in the system throughout the simulation.

After the NVE, instead of continuing the simulations on the final conformation, clustering was conducted on all the conformations observed during the MD using GROMACS. A cutoff of $0.10-0.20 \mathrm{~nm}$ was used for clustering analysis ${ }^{53}$. Further simulations were continued on the most favorable conformation, i.e. frequency was $60 \%$ or more during the NVE. We extended the MD runs out to $100 \mathrm{~ns}$ to verify the equilibrium and we observed similar results (Results not shown). Once the most favorable conformation was defined, a water box was generated that was large enough to conduct the steered molecular dynamics (SMD). Minimization, heating, NPT ensemble and NVE ensemble were conducted on the system again following the 
same procedure as before, while the position of the protein was fixed to prepare the system for SMD. Finally, SMD pulling was carried out on the protein where the $\mathrm{N}$-terminus of the motif (the amide group attached to the alpha carbon of the first amino acid) was held fixed while pulling on the C-terminus (the carboxyl group attached to the alpha carbon of the final amino acid) at a constant velocity of $0.1 \mathrm{~m} / \mathrm{s}$. This velocity was determined by running simulations on three different motifs at different velocities $(0.1,0.5,1,5,10$ and $50 \mathrm{~m} / \mathrm{s})$. Although $0.1,0.5$ and $1 \mathrm{~m} / \mathrm{s}$ gave similar results, the velocity chosen for the $\mathrm{SMD}$ was $0.1 \mathrm{~m} / \mathrm{s}$, because according to Gautieri et al. $^{41}$, modulus converges to constant value for pulling velocity less than $0.5 \mathrm{~m} / \mathrm{s}$.

The extended structure after SMD was put into a water box and the MD simulation was continued by releasing the N- and Ctermini for a total $60 \mathrm{~ns}$ to compare the collapsed structure with the structure obtained after clustering. Structures were aligned using the Chimera 1.9 visualization program ${ }^{54}$.

Secondary structure assignments were performed using the Xtlsstr program ${ }^{55}$. Even though DSSP ${ }^{56}$ is a widely used program, XtIsstr was chosen due to its ability to identify polyproline II (PPII) type helices in addition to other secondary structures. The simulations were repeated to determine the effect of entropy on the protein. The force field was modified by switching off the attractive part of the Lennard-Jones (LJ) interactions (C6) and all electrostatic interactions, leaving only the repulsive part of the Lennard-Jones interactions $(\mathrm{C} 12)$ and bonded interactions as described by Grater et al. ${ }^{57}$. The resulting model is only restricted by local steric repulsion of the atoms and the bonded interactions, mainly the dihedral potentials along the protein backbone. Hydrogen bonds within the motif were predicted using the molecular visualization software RasTop 2.2 (http://www.geneinfinity.org/rastop/).

\subsection{Results and Discussion}

\subsubsection{Equilibrium}

Analysis of unrestrained MD simulations based on root-mean-square deviation (RMSD) revealed that the systems changed quickly from their initial structures but then remained relatively unchanged out to 100 ns (supplementary information, Fig. S1). Although substantial fluctuations were observed since these peptides are relatively small and disordered, systematic changes in RMSD were not apparent.

\subsubsection{Clustering}

A clustering analysis ${ }^{53}$ of the MD simulations was conducted based on the backbone atoms to determine the most favorable conformation within each simulation. During the MD simulation, conformations were recorded at every 500 time-steps, followed by clustering on all the conformations. Using a cutoff of $0.15-0.20 \mathrm{~nm}$, frequencies of the most favorable conformational cluster were 61\% for PSSSYGAPGGGNGGR, 59\% for PSSSYSAPGGGNGGR, 66\% for PSSTYGAPGGGNGGR and 74\% for PSSTYSAPGGGNGGR. The latter peptide is not found among reported sequences for natural resilin but is a composite 
synthetic sequence representing both the SYSAP and TYGAP variants of SYGAP. The representative structure for each peptide from the clustering is shown in Fig. 1. Notably, due to the bend between tyrosine (TYR) and alanine (ALA) for PSSSYGAPGGGNGGR, the end-to-end distance of this motif (20尺̊) was about one-half that of the other motifs (Table 1).

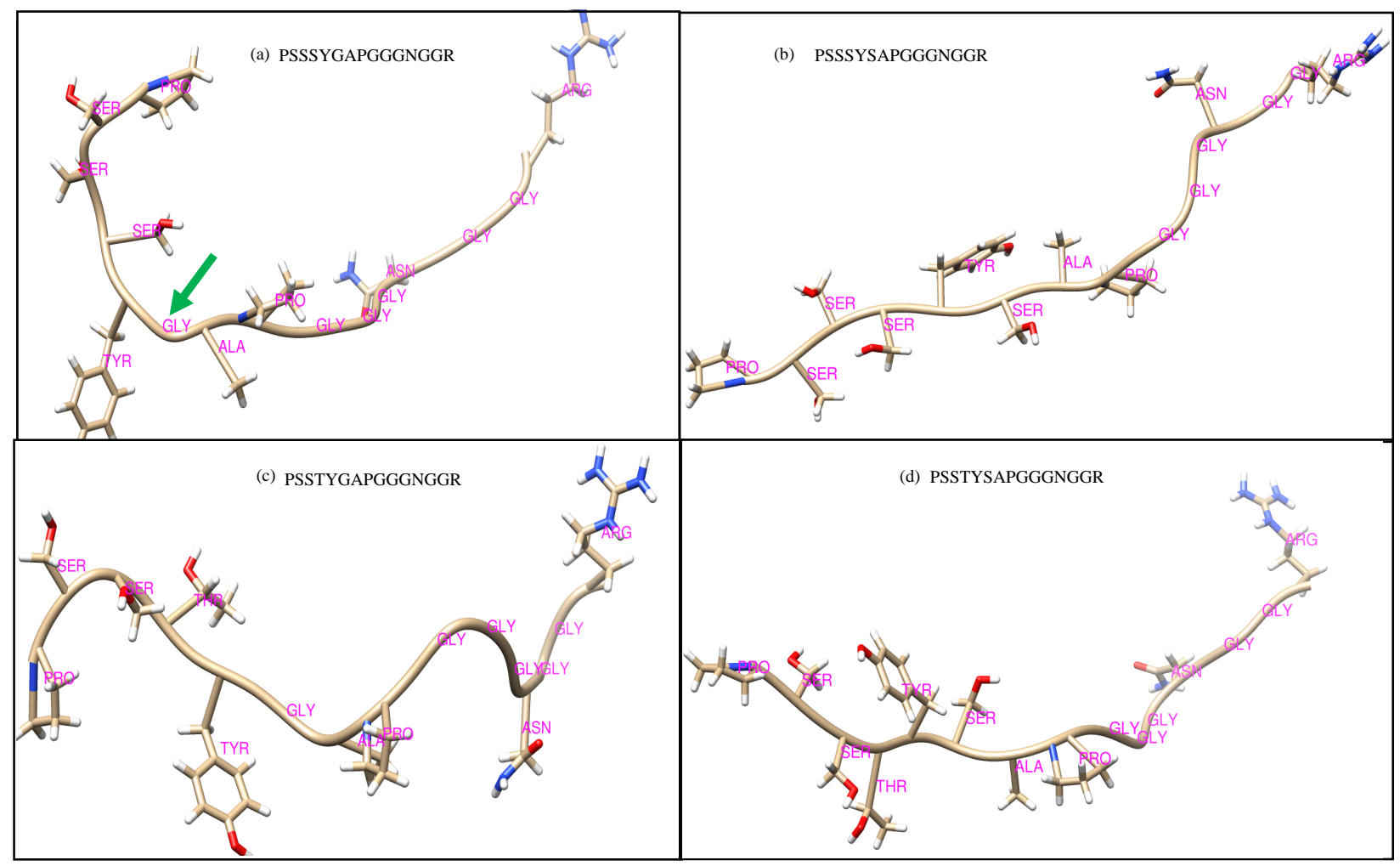

Fig. 1 Structure of the four peptides after clustering of MD results (a) PSSSYGAPGGGNGGR, (b) PSSSYSAPGGGNGGR, (c) PSSTYGAPGGGNGGR and (d) PSSTYSAPGGGNGGR. Due to the bend between tyrosine (TYR) and alanine (ALA) for PSSSYGAPGGGNGGR, at arrow in (a), the end-to-end distance of the motif is smaller $\left(20 \mathrm{~A}^{\circ}\right)$ compared to the other motifs.

Table 1. Energies and non-bonded interactions between protein and water of the repeating motifs. Force at $55 \%$ strain for every motif is recorded. The most common repeating motif of Drosophila melanogaster, PSSSYGAPGGGNGGR, has the highest extensibility and least stiffness compared to others.

\begin{tabular}{|c|c|c|c|c|c|c|c|}
\hline Fragments & $\begin{array}{c}\text { Bond } \\
\text { Energy } \\
(\mathrm{kcal} / \mathrm{mol})\end{array}$ & $\begin{array}{c}\text { van der } \\
\text { Waals } \\
\text { Energy } \\
(\mathrm{kcal} / \mathrm{mol})\end{array}$ & $\begin{array}{c}\text { Total } \\
\text { Energy } \\
(\mathrm{kcal} / \mathrm{mol})\end{array}$ & $\begin{array}{c}\text { Potential } \\
\text { Energy } \\
(\mathrm{kcal} / \mathrm{mol})\end{array}$ & $\begin{array}{c}\text { Force } \\
\text { at } 55 \% \\
\text { strain } \\
(\mathrm{pN})\end{array}$ & $\begin{array}{c}\text { non- } \\
\text { bonded } \\
\text { interaction } \\
\text { between } \\
\text { protein \& } \\
\text { water, } \\
(\mathrm{kcal} / \mathrm{mol})\end{array}$ & $\begin{array}{c}\text { Length at } \\
\text { Equilibrium } \\
(\AA)\end{array}$ \\
\hline PSSSYGAPGGGNGGR & 5500 & 7800 & -41430 & -53710 & $\mathbf{2 2}$ & -710 & $\mathbf{2 0 . 6 8}$ \\
\hline PSSSYSAPGGGNGGR & 6860 & 9850 & -52300 & -67540 & 2902 & -700 & 40.04 \\
\hline PSSTYGAPGGGNGGR & 7070 & 10130 & -53870 & -69630 & 2250 & -690 & 38.33 \\
\hline PSSTYSAPGGGNGGR & 7960 & 11450 & -60800 & -78460 & 1620 & -710 & 37.68 \\
\hline
\end{tabular}




\subsubsection{Pulling Velocity Influenced the Force Required for Displacement}

Using the representative structures from the unrestrained MD simulations, pulling simulations were conducted. The effect of pulling velocity, over a range including $v=50,10,5,1,0.5$, and $0.1 \mathrm{~m} / \mathrm{s}$, was examined initially. Using the peptide PSSSYSAPGGGNGGR, there are only small qualitative differences in the forces at velocities between $1,0.5$, and $0.1 \mathrm{~m} / \mathrm{s}$, but there are notable differences for the higher velocities (Fig. 2). Similar results were also obtained for other peptides. This effect of pulling velocity on force is similar to that obtained for collagen by Kwansa et al. ${ }^{36}$ and Gautieri et al. ${ }^{40}$. According to Gautieri et al. $^{40}$, the apparent modulus of tropocollagen converges to a constant value for pulling rates below $0.5 \mathrm{~m} / \mathrm{s}$ because force vs strain plots are similar for $0.5 \mathrm{~m} / \mathrm{s}$ or lower pulling rates. These similar outcomes from pulling simulations of different peptides may result from compatible interactions between the amino acids and their surroundings. In our studies, bond energy, van der Waals energy, potential energy, total energy, and non-bonded interaction energy between water and protein were almost identical for all the velocities, which implied these energies do not make much contribution to the variations at different velocities.

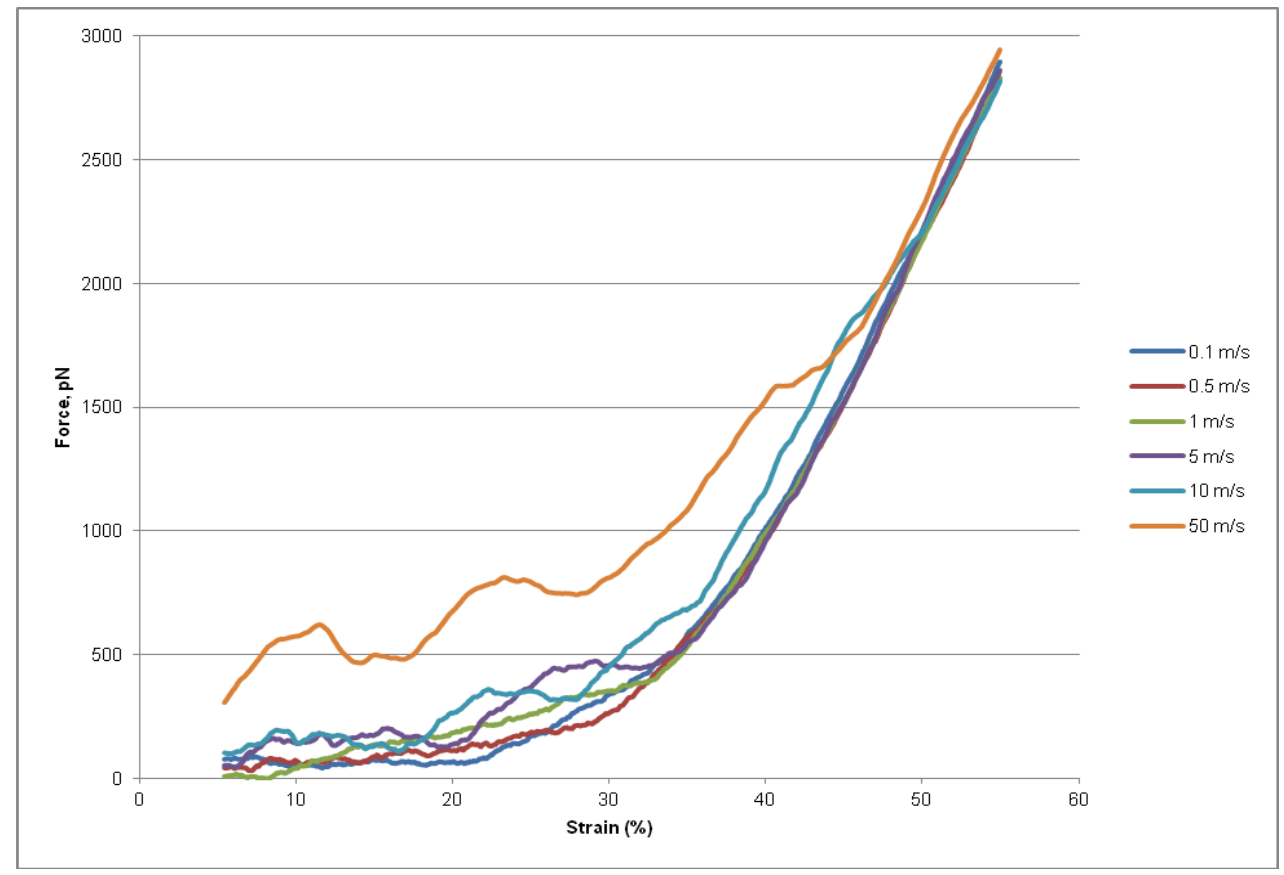

Fig. 2 Force vs Strain plot for different pulling velocitites on peptide PSSSYSAPGGGNGGR. Strain represents engineering strain of the $40.04 \AA$ long molecular model and was defined as the change of length over the initial length (end-to-end distance). 


\subsubsection{Repeating Motifs in Exon-1}

The Drosophila exon 1 resilin domain has PSSSYGAPGGGNGGR as the most common repetitive motif ${ }^{30}$. During our work, we have considered four sequence motifs PSSSYGAPGGGNGGR, PSSTYGAPGGGNGGR, PSSSYSAPGGGNGGR and PSSTYSAPGGGNGGR to understand whether the most frequent motif has any significance over others. From the force versus strain curves obtained from the repeating motifs shown in Fig. 3, the PSSSYGAPGGGNGGR motif has lowest stiffness. The force required to pull this motif is very small compared to other motifs for equal strain. The fluctuations of force for PSSSYGAPGGGNGGR are probably due to the interactions with the surrounding water and among the amino acids. Similar fluctuations can be obtained for all the plots in Fig. 3, if all of them are plotted at the same scale (supplementary information, Fig. S2). Results show that the force required to deform $55 \%$ of the initial end-to-end distance is much smaller for the most common repetitive motif compared to others as shown in Table 1.

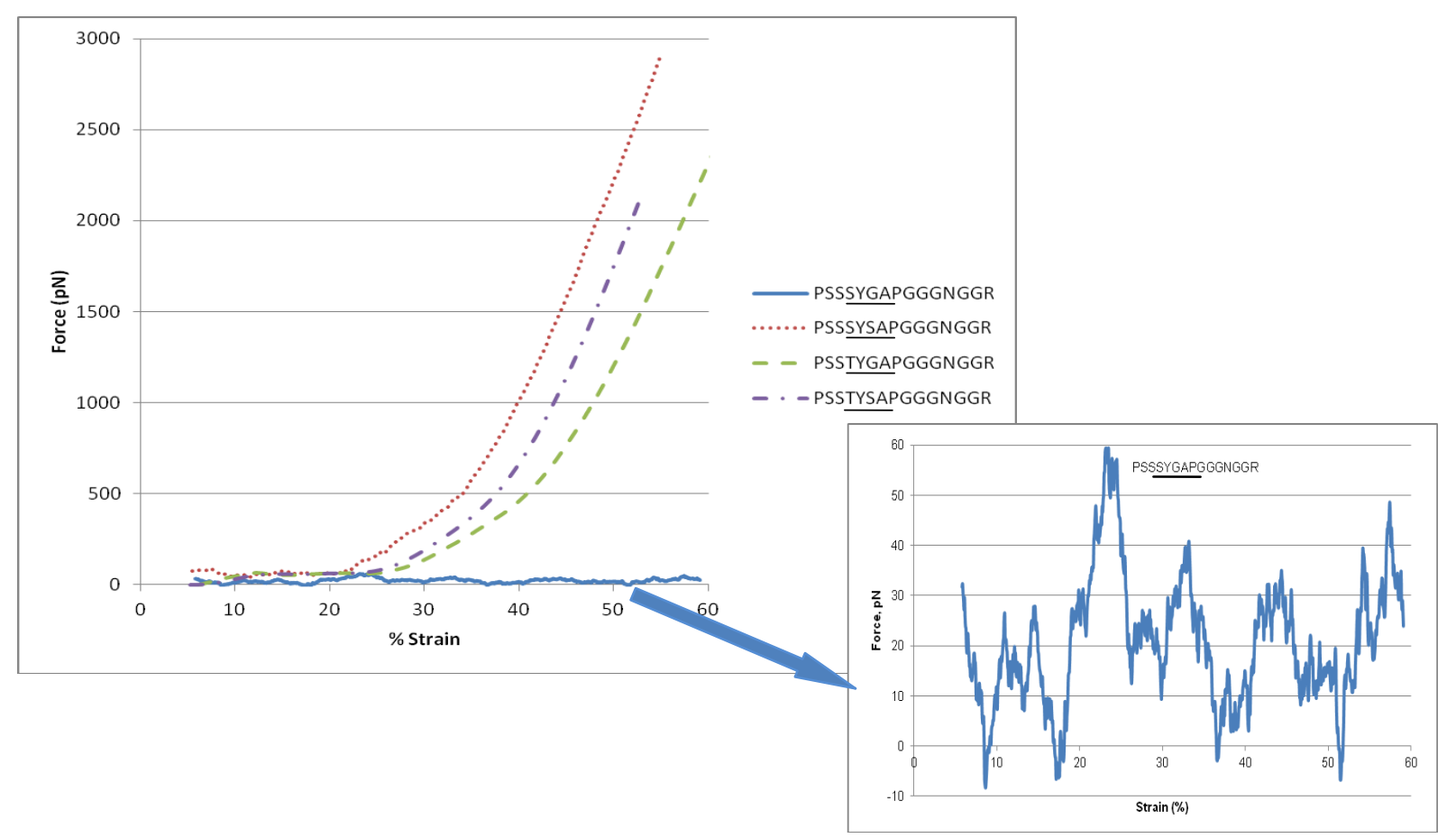

Fig. 3 Force-displacement plot for PSSSYGAPGGGNGGR, PSSSYSAPGGGNGGR, PSSTYGAPGGGNGGR and PSSTYSAPGGGNGGR. Among the motifs, PSSSYGAPGGGNGGR has highest extensibility and this is the most frequently represented repetitive motif in all insect resilin (Inset figure is provided at smaller scale). The Fluctuations are probably due to the interactions with the surrounding water and among the amino acids. 
Referring back to the structures in Fig. 1, we see that PSSSYGAPGGGNGGR has the smallest length (Table 1), which results from a bend at the glycine residue between tyrosine and alanine (Fig. 2a). This bend may be favored not only by the lack of a side chain on glycine, but also due to the presence of serine before tyrosine instead of threonine which has an extra methyl group which can create steric hindrance. The MD simulation was repeated three times and the same bend at the glycine was observed each time. All of the other motifs have a bend at the GGGN and GGR building blocks, but those bends do not influence the end-to-end distance to the same extent, as is shown in Table 1. Bent motifs could offer increased flexibility over more linearly arranged motifs if the bend can change shape when loaded, and if this elongated motif can come back to its initial bent conformation when force is removed, then this bent conformation may contribute to the macroscopic elastomeric properties.

\subsubsection{Secondary Structure Analysis}

Resilin is a disordered elastomeric protein in which there is no secondary structure like $\alpha$-helix or $\beta$-sheet. However, resilin may possess polyproline II (PPII) helices ${ }^{4}$ or $\beta$-turns ${ }^{1,29}$ which may influence its properties. According to Teule et. al. ${ }^{58}$, central PG residue in a consensus motif favors the formation of type II $\beta$-turns. We have examined all four motifs using Xtlsstr software for PPII helix and results are shown in Table 2. The most frequent motif, PSSSYGAPGGGNGGR, has the fewest number of amino acids participating to form PPII, which might influence formation of a bent structure because these amino acids will not participate to form secondary structure.

Table 2. Polyproline II (PPII) helix in the four motifs. PSSSYGAPGGGNGGR has fewest numbers of amino acids that can adopt phi, psi values characteristic of PPII helix. Both PSSSYSAPGGGNGGR and PSSTYSAPGGGNGGR also have $\beta$-strand structure in addition to PPII while PSSTYGAPGGGNGGR only has PPII. Lowercase letters in the Xtlsstr assignment denote the end residues of secondary structure stretches.

\begin{tabular}{|c|c|c|c|}
\hline Sequence & PPII & Pattern $^{\text {a }}$ & Location $^{-1}$ \\
\hline PSSSYGAPGGGNGGR & 2 & ----- Pp------ & 7,8 \\
\hline PSSTYGAPGGGNGGR & 4 & $--P P p-P p------$ & $3,4,5,7,8$ \\
\hline PSSSYSAPGGGNGGR & 5 & $---P p E e----P p--$ & $4,5,12,13$ \\
\hline PSSTYSAPGGGNGGR & 4 & EEe-PPPP-NNN--- & $5,6,7,8$ \\
\hline
\end{tabular}

${ }^{a}$ Secondary structures disordered (-, N); poly-proline II-helix (P, p); $\beta$-strand (E, e)

\subsubsection{Effects of building blocks}

Exon-1 of resilin from D. melanogaster has PSSSYGAPGGGNGGR as the most frequent repetitive motif ${ }^{30}$. This motif can be subdivided into small building blocks: PSS, SYGAP, GGGN and GGR. Among the building blocks, most insects have SYGAP though some also have SYSAP and TYGAP. To gain further insight regarding the aforementioned bend in PSSSYGAPGGGNGGR, which resulted in a much shorter end-to-end distance compared to other motifs, and to determine whether the flanking building 
blocks (PSS, GGGN and GGR) influence the bend, we analyzed the following partial versions of the resilin pentadecapeptide: SYGAP, PSSSYGAP, PSSSYGAPGGGN. Similar building block combinations were constructed for all variations of the repetitive motifs under investigation here. MD simulations yielded results comparable to those for the full-length pentadecapeptide, i.e. motifs containing the repeat SYGAP exhibited the characteristic bending between tyrosine $(\mathrm{Y})$ and alanine $(\mathrm{A})$, and end-to-end distance is smaller compared to motifs with SYSAP, TYGAP and TYSAP (Table 3). As building blocks PSS, GGGN and GGR had very little influence on the bend, we reasoned that a further comparison of SYGAP, SYSAP, TYGAP and TYSAP might yield new insight on the diverse functions of resilins and the distinct bend in SYGAP-containing peptides.

Table 3. End-to-end distances of fragments of the resilin motif comprised of varying building blocks. End-to-end distances of fragments containing SYGAP (in bold) are always the shortest within each fragment class.

\begin{tabular}{|c|c|c|c|c|c|}
\hline Fragments & $\begin{array}{c}\text { End-to end } \\
\text { distance }(\AA)\end{array}$ & Fragments & $\begin{array}{c}\text { End-to end } \\
\text { distance }(\AA)\end{array}$ & Fragments & $\begin{array}{c}\text { End-to end } \\
\text { distance }(\AA)\end{array}$ \\
\hline SYGAP & $\mathbf{4 . 8 5}$ & PSSSYGAP & $\mathbf{8 . 2 8}$ & PSSSYGAPGGGN & $\mathbf{1 1 . 3 8}$ \\
\hline SYSAP & 12.01 & PSSSYSAP & 17.14 & PSSSYSAPGGGN & 20.81 \\
\hline TYGAP & 14.25 & PSSTYGAP & 21.29 & PSSTYGAPGGGN & 23.89 \\
\hline TYSAP & 15.4 & PSSTYSAP & 24.55 & PSSTYSAPGGGN & 30.79 \\
\hline
\end{tabular}

We have calculated all energy contributions and interaction energies between peptide and water, as shown in Table 4. All the energies for the various repeating fragments are almost the same. The only difference among these four fragments is their length after equilibrium and before SMD. The length of SYGAP is $4.852 \AA$, which is less than half of the length of the other fragments. We performed the MD simulations three times with identical results. Among the four fragments, SYGAP and TYGAP have glycine in the central position, which provides conformational freedom for protein folding, but TYGAP has threonine instead of serine at the beginning of this sequence. The larger size of the threonine side chain may produce steric hindrance that disfavors the bending seen for SYGAP. On the other hand, SYSAP and TYSAP do not contain glycine, so they cannot bend like SYGAP. Our synthetic composite peptide assembled for this study, TYSAP, has both threonine and serine. Its higher steric hindrance than the naturally occurring peptides may explain both its lack of bending and possibly its apparent absence from the repertoire of naturally occurring resilins. 
Table 4. Energies and non-bonded interactions between peptide and water of the repeating fragments. Force at $1 \mathrm{~nm}$ elongation for every fragment is recorded. For all four repeating fragments, Forces at $1 \mathrm{~nm}$ strain and length at equilibrium have noticeable variation as shown in the table.

\begin{tabular}{|c|c|c|c|c|c|c|c|}
\hline Fragments & $\begin{array}{c}\text { Bond } \\
\text { Energy } \\
(\mathrm{kcal} / \mathrm{mol})\end{array}$ & $\begin{array}{c}\text { Van-Der } \\
\text { Waals } \\
\text { Energy } \\
(\mathrm{kcal} / \mathrm{mol})\end{array}$ & $\begin{array}{c}\text { Total } \\
\text { Energy } \\
(\mathrm{kcal} / \mathrm{mol})\end{array}$ & $\begin{array}{c}\text { Potential } \\
\text { Energy } \\
(\mathrm{kcal} / \mathrm{mol})\end{array}$ & $\begin{array}{c}\text { Force } \\
\text { at } 1 \mathrm{~nm} \\
\text { strain } \\
(\mathrm{pN})\end{array}$ & $\begin{array}{c}\text { non- } \\
\text { bonded } \\
\text { interaction } \\
\text { between } \\
\text { protein \& } \\
\text { water, } \\
(\mathrm{kcal} / \mathrm{mol})\end{array}$ & $\begin{array}{c}\text { Length at } \\
\text { Equilibrium } \\
(\AA)\end{array}$ \\
\hline SYGAP & 4990 & 5780 & -28350 & -40470 & 147 & -408 & $\mathbf{4 . 8 5 2}$ \\
\hline SYSAP & 4960 & 5800 & -28630 & -40620 & 2220 & -416 & 12.01 \\
\hline TYGAP & 5000 & 5840 & -28640 & -40721 & 3678 & -372 & 14.25 \\
\hline TYSAP & 5080 & 5910 & -29020 & -41331 & 5732 & -422 & 15.40 \\
\hline
\end{tabular}

Ramachandran plots ${ }^{59}$ are used frequently to look at the distribution of the dihedral angles between the amino acids ${ }^{60,61}$ and the effect of individual amino acids on the secondary structure of the proteins ${ }^{62-63}$. We have compared the dihedral angles during the MD for SYGAP, SYSAP, TYGAP and TYSAP using the Ramachandran plot at the bending location (glycine (G) for SYGAP and TYGAP, and serine (S) for SYSAP and TYSAP). We observed that the glycine of SYGAP does not have any preferred structure while the others have preferred ordered structures as shown in Fig. 4, which indicates the greater tendency of SYGAP to form disordered structure relative to the other peptides tested here. Theoretically, glycine might be in a BII turn conformation $\left(\phi_{3}=90\right.$ and $\psi_{3}=0$, where $\phi_{3}$ and $\psi_{3}$ are the third residue of the turn as stated by Richardson ${ }^{63}$ and Li at. al. $^{34}$ ). From Fig. 4, we can see that the glycine of SYGAP has the tendency to form a $\beta I I$-turn which is one of the characteristics of disordered proteins ${ }^{1}$, while none of the other amino acids at the same location showed a similar tendency. 


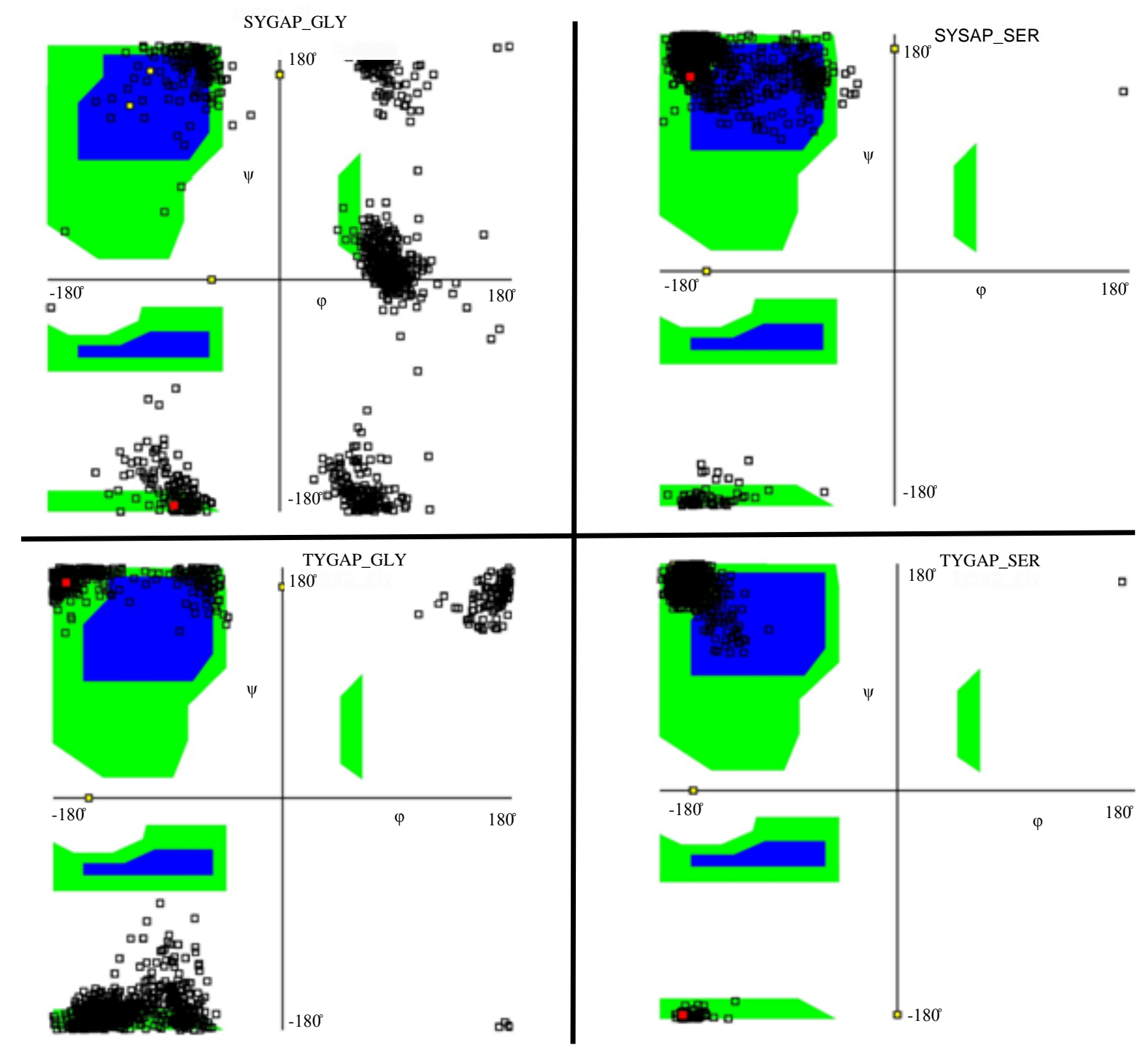

Fig. 4 Ramachandran plots for the fragments SYGAP, SYSAP, TYGAP and TYSAP during the MD at the third amino acid, which corresponds to the bending location of SYGAP.

The presence of a bend in SYGAP will be significant, if it can be extended and then return to its initial bent conformation when force is removed. To test this possibility, we conducted a MD simulation for 60 ns on the elongated structure of SYGAP after steered molecular dynamics (SMD), i.e. on the $1 \mathrm{~nm}$ elongated structure. Results showed that the structure of the backbone of SYGAP after unrestrained MD of the extended peptide has a structure very similar to that of the starting structure prior to extension (Fig. 5). The backbone RMSD of these structures is $0.26 \AA$. 


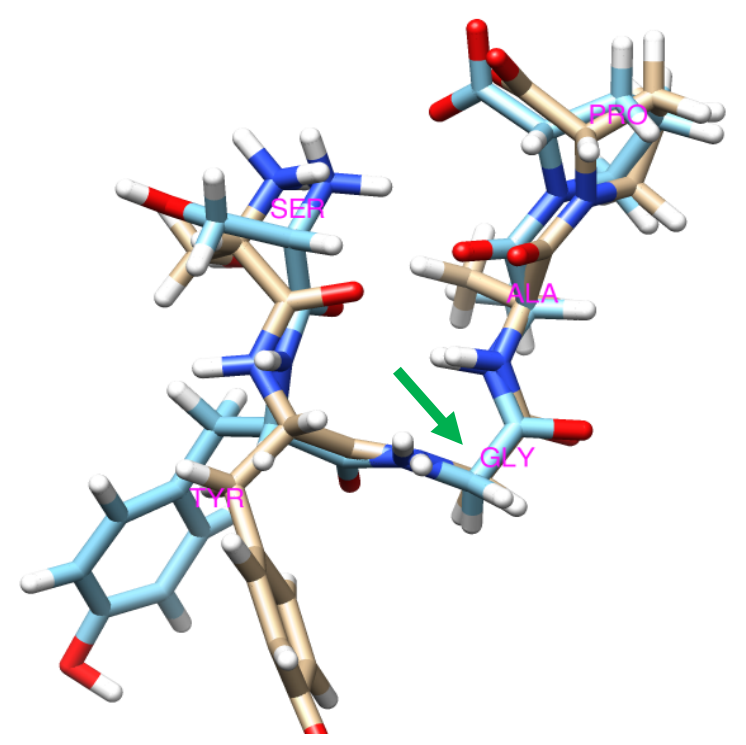

Fig. 5 Alignment of SYGAP structure obtained before extension by SMD (tan) and after collapse during unrestrained MD for $60 \mathrm{~ns}$ (blue). Both of structures have a similar bend at glycine (arrow).

To determine the entropically preferred conformation, we switched off the electrostatic interactions and the attractive portion of the Lennard-Jones ( $\amalg)$ interactions leaving only bonded interactions and the repulsive part of the $\amalg$ interactions ${ }^{57}$. From the results, we observed that SYGAP has a comparable bent structure (Fig. 6), although the length (8.215 $\AA$ ) is longer than when electrostatic interactions are present (Table 4) possibly reflecting an influence on secondary structure. On the other hand, the end-to-end distances observed for TYGAP, SYSAP and TYSAP, at 12.16, 11.67 and $13.94 \AA$, respectively, were similar to those in the presence of all the force field terms (Table 4). Cheng et al. ${ }^{6}$ conducted a similar study on spider silk and resilin, and showed a comparably low persistence length due to the low steric hindrance of glycines. Moreover, Dicko et al. ${ }^{64}$ concluded that the emergence of elasticity in silks correlates highly with the glycine content. They suggested that glycine controls the soluble precursor assembly and proline governs the solid fiber behavior. According to our results, SYGAP forms a bent structure, and end-to-end distance is very short compared to the other repeats which agrees with result from Cheng et al. $^{6}$, as stated above. Resilin also has high glycine content like other elastomeric proteins and here we show that a characteristic bend occurs at the glycine of the SYGAP repeat, which may be one of the controlling factors for high elasticity. 


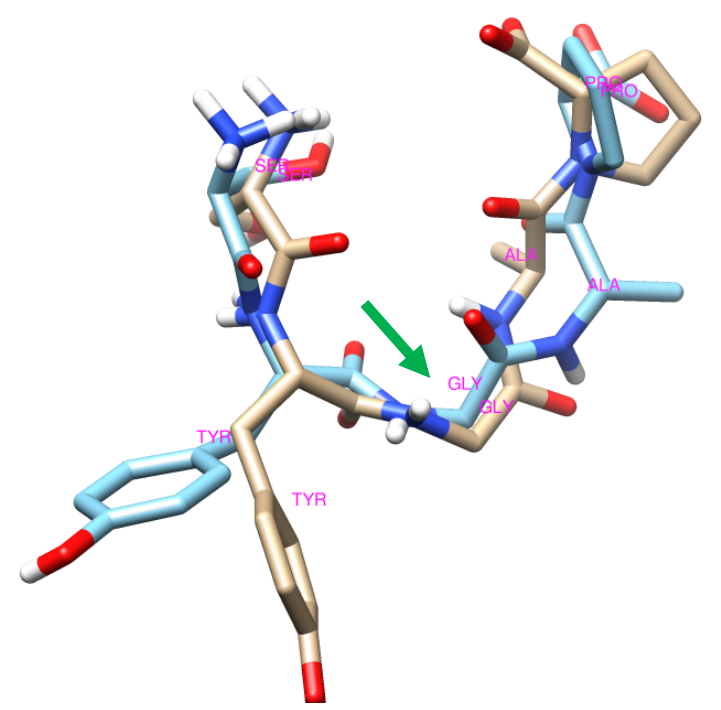

Fig. 6 Alignment of SYGAP structure obtained before (tan) and after switching off the electrostatic interactions and the attractive portion of the Lennard-Jones (LJ) interactions (blue). Both structures have a similar bend at the glycine residue (arrow).

We have also constructed another fragment, GYGAP to test steric hindrance, as this fragment has the least steric hindrance compared to any of the other four fragments due to the absence of side chains. The end-to-end distance for GYGAP is $14.8 \AA$, which is comparable to the length of SYSAP, TYGAP and TYSAP. These results indicate that bent and extended structures resulting for these fragments at equilibrium are not only from steric hindrance, but also from the electrostatic interactions.

To clarify the possibility of charged terminal interactions between serine $(\mathrm{S})$ and proline $(\mathrm{P})$ in the bent conformation of SYGAP, we attached caps at both the termini. The resulting MD simulation also gave results comparable to those obtained without capping the termini (supplementary information, Fig. S3).

From the dihedral angle results we saw that SYGAP does not have any preferred structure while the other three peptides, SYSAP, TYGAP and TYSAP, prefer some secondary structure (Table 2). When we consider electrostatic interactions, there are possibilities of hydrogen bonds formation in the chain. However, due to steric hindrance some of the possible $\mathrm{H}$ bonds cannot be formed. During the MD simulation, the backbone rotates through different dihedral angles, as shown in the Ramachandran plots, and due to the different side groups, the peptides switch among different conformations. During the process, some residues form hydrogen bond and construct stable and preferred conformations. According to Johnson et. al. ${ }^{65}$, an enhancement in the modulus elasticity and toughness occurs due to the tyrosine and serine stabilization through hydrogen bonding. SYGAP has four H-bonds within the backbone and the side chains, and forms the bent conformation shown in Fig. 7. However, for SYSAP, TYGAP and TYSAP, only one H-bond within the backbone and the side chains can be formed. According to 
Rauscher et. al. ${ }^{1}$, if the sequence has a higher propensity of PPII structure, the structure cannot stabilize any turns through hydrogen bonding. As a result SYSAP, TYGAP and TYSAP do not have any stable bent conformations.

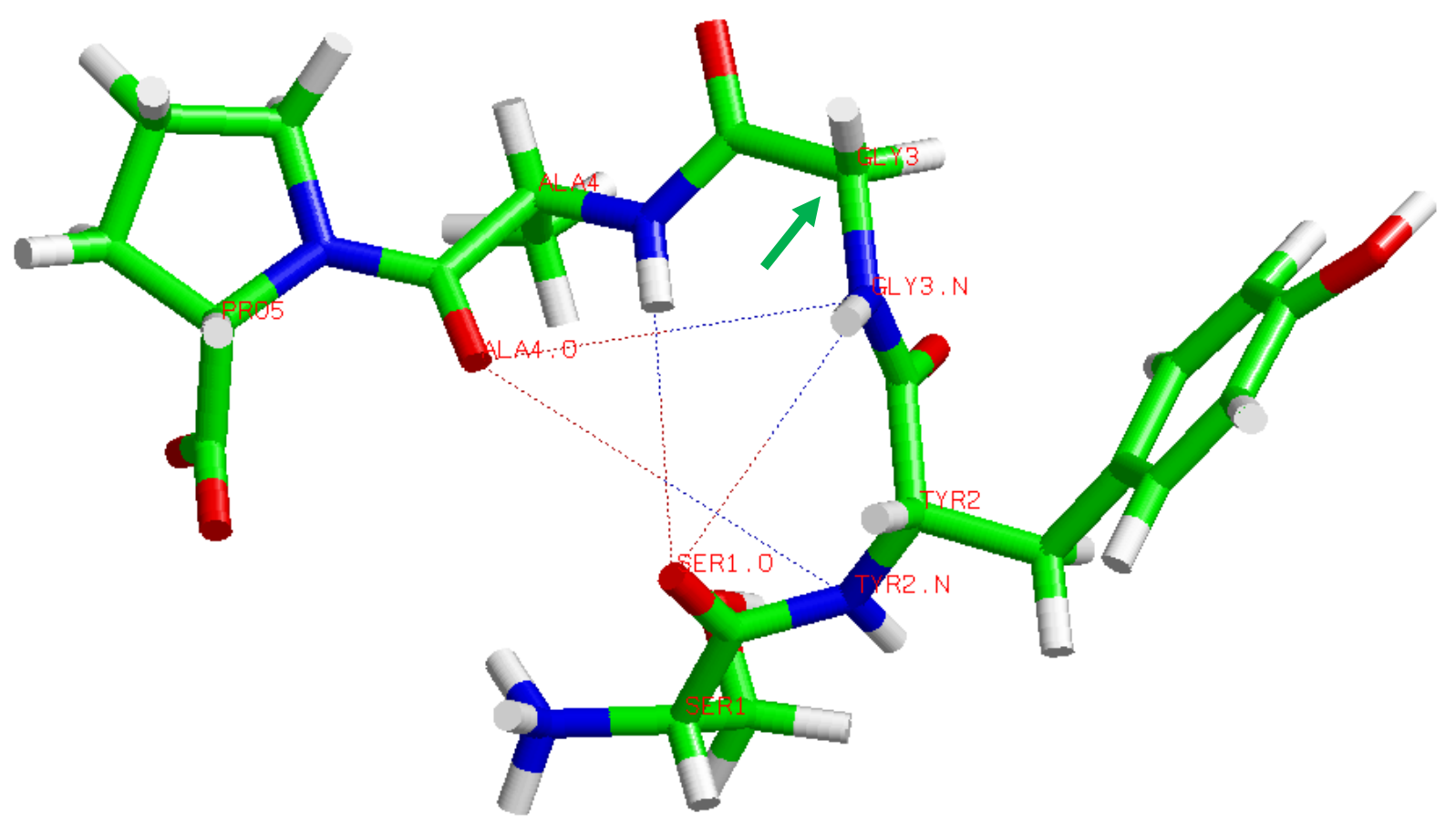

Fig. 7 Hydrogen bonds in the repeat motif SYGAP during bent conformation. Other than proline, all the other amino acids participate in hydrogen bonding to form the stable bent structure in addition to lack of streric hindrance at glycine (arrow).

We have also tested the order of the amino acids in the SYGAP to understand if there is any significance. We have switched alanine and proline and formed SYGPA. The MD simulation results showed that SYGPA behaves similarly to TYGAP, SYSAP and TYSAP, i.e. forming an extended structure. It does not form the bent structure and there is only one hydrogen bond within the backbone and the side chains.

Together our results showed that the most frequent naturally occurring motif, PSSSYGAPGGGNGGR, adopts conformations that may contribute to improved elastomeric behavior compared to other related motifs. Importantly, PSSSYGAPGGGNGGR forms a bent structure, such that when we hold one end and pull the other end, the peptide unfolds, requiring very low forces to stretch in comparison to the other tested variations of this motif. When we remove the force from the PSSSYGAPGGGNGGR, it returns to its previous conformation, as was demonstrated by the collapse simulation (Fig. 5). From these results, we hypothesize that this ability to bend might be one of the contributing factors of the elastomeric properties of resilin. 


\subsection{Conclusion}

Disordered elastic proteins are found throughout the animal kingdom for a variety of functions. Like other disordered elastomeric proteins (gliadin, elastin, spider silks), resilin contains distinct repetitive domains in its amino acid sequence to confer elastomeric properties $^{30}$. In exon 1, PSSSYGAPGGGNGGR is the most frequent repetitive motif. Our objective was to determine if this repeating motif has any unique properties over other possible motifs and how this motif may thereby control the behavior of the elastomeric proteins. The results suggest that this motif makes an important contribution to the elastomeric properties of resilin by forming a favorable bent structure instead of an extended structure through a combined effect of $\mathrm{H}$-bonding and lack of steric hindrance. When the protein is stretched, it moves to an extended conformation, and it comes back to the initial conformation when released due to the favorable bend. What we learned from simulating the repetitive motifs of resilin may be applicable to the biology and mechanics of other unstructured protein domains. These findings might someday be valuable in the design and manufacture of high-performance, biocompatible rubbers with tailored mechanical properties. Moreover, we anticipate that the results will be of scientific significance in the field of biomaterials and biomedical devices.

\section{Acknowledgements}

We thank Dr. David Bevan's graduate students Anne M. Brown and former ESM graduate student Albert L. Kwansa, for their help with the use of GROMACS and NAMD. We also thank the Biomedical Engineering and Mechanics (BEAM) Department for use of the Linux Computer Cluster and Virginia Tech for providing us with computational resources on the ARC Blueridge computer. 


\section{References}

1. Rauscher, S., Baud, S., Miao, M., Fred W. Keeley, F. W., and Pomes, R. (2006) Proline and Glycine Control Protein SelfOrganization into Elastomeric or Amyloid Fibrils, Structure 4, 1667-1676.

2. Tompa, P. (2002) Intrinsically unstructured proteins, Trends Biochem Sci. 269, 2-12.

3. Bochicchio, B., Pepe, A., and M., T. A. (2008) Investigating by CD the molecular mechanism of elasticity of elastomeric proteins., Chirality 20, 985-994.

4. Theillet, F. X., Kalmar, L., Tompa, P., Han, K. H., Selenko, P., A. Keith Dunker, Daughdrill, G. W., and Uversky, V. N. (2013) The alphabet of intrinsic disorder: I. Act like a Pro: On the abundance and roles of proline residues in intrinsically disordered proteins, Intrinsically Disordered Proteins 1, 1-13.

5. Williams, R. M., Obradovi, Z., Mathura, V., Braun, W., Garner, E. C., Young, J., Takayama, S., Brown, C. J., and Dunker, A. K. (2001) The protein non-folding problem: amino acid determinants of intrinsic order and disorder, Pac Symp Biocomput 89100 .

6. Cheng, S., Cetinkaya, M., and Grater, F. (2010) How Sequence Determines Elasticity of Disordered Proteins, Biophysical Journal 99, 3863-3869.

7. Weis-Fogh, T. (1960) A rubber-like protein in insect cuticle, Journal of Molecular Biology, 889-907.

8. Weis-Fogh, T. (1961) Molecular interpretation of the elasticity of resilin, a rubber-like protein, Journal of Molecular Biology 3, 648-667.

9. Jensen, M., and Weis-Fogh, T. (1962) Biology and Physics of Locust Flight V. Strength and Elasticity of Locust Cuticle, Phil. Trans. R. Soc. Lond. B 245, 137-169.

10. Lyons, R. E., Lesieur, E., Kim, M.,Wong, D.C.C., Huson, M.G., Nairn, K. M., Brownlee, A. G., Pearson, R. D., Elvin, C. M. (2007) Design and facile production of recombinant resilin-like polypeptides: gene construction and a rapid protein purification method, Protein Engineering, Design \& Selection 20, 25-32.

11. Elvin, C. M., Carr, A. G., Huson, M. G., Maxwell, J. M., Pearson, R. D., Vuocolo, T., Liyou, N. E., Wong, D. C. C., Merritt, D. J., and Dixon, N. E. (2005) Synthesis and properties of crosslinked recombinant pro-resilin, Nature 437, 999-1002.

12. Lv, S., Dudek, D., Cao, Y., Balamurali, M. M., Gosline, J. and Li, H. (2010) Designed biomaterials to mimic the mechanical properties of muscles, Nature 465, 69-73.

13. Edwards, S. J. (1960) Predation and digestion in assassin bugs (Heteroptera, Reduviidae, University of Cambridge, UK.

14. Young, D., and Bennet-Clark, H. C. (1995) The role of tymbal in cicada sound production, J. Exp. Biol. 198, 1001-1019.

15. Burrows, M., Shaw, S. R., and Sutton, G. P. (2008) Resilin and chitinous cuticle form a composite structure for energy storage in jumping by froghopper insects, BMC Biology 6.

16. Andersen, S. O., and Weis-Fogh, T. (1964) Resilin. A rubber-like protein in arthropod cuticle., Adv. Insect Physiol. 2, 1-65.

17. Bennet-Clark, H. C., Daws, A. G. (1999) Transduction of mechanical energy into sound energy in the cicada Cyclochila australasiae., The Journal of Experimental Biology 202, 1803-1817.

18. Skals, N., Surlykke, A. (1999) Sound production by abdominal tymbal organs in two moth species: the green silver-line and the scarce silver-line (Noctuoidea: Nolidae: Chloephorinae). Journal of Experimental Biology 202, 2937-2949.

19. Neff, D., S. F. Frazier. (2000) Identification of resilin in the leg of cockroach, Periplaneta americana: confirmation by a simple method using pH dependence of UV fluorescence., Arthropod Structure \&amp; Development 29, 75-83.

20. Kram, R., B. Wong. (1997) Three-dimensional kinematics and limb kinetic energy of running cockroaches., Journal of Experimental Biology 200, 1919-1929.

21. Bennet-Clark, H. C., and Lucey, E. C. A. (1967) The Jump of the Flea: A Study of the Energetics and a Model of the Mechanism., The Journal of Experimental Biology 47, 59-76.

22. Patek, S. N., Dudek, D. M., and Rosario, M. V. (2011) From bouncy legs to poisoned arrows: elastic movements in invertebrates, The Journal of Experimental Biology 214, 1973-1980.

23. Haas, F., Gorb, S., and Wootton, R. J. (2000) Elastic joints in dermapteran hind wings: materials and wing folding., Arthropod Struct. Develop. 29, 137-146.

24. Elliott, G. F., Huxley, A. F., and Weis-Fogh, T. (1965) On the structure of resilin, Journal of Molecular Biology 13, 791-795, IN711-IN713.

25. Kappiyoor, R., Balasubramanian, G., Dudek, D. M., Puri, I.P. (2011) Elastomechanical properties of resilin, Soft Matter 7, 11006-11009.

26. Petrenko, R. (2010) Computer simulations of resilin-like peptides, In Physics, University of Cincinnati.

27. Andersen, S. O. (1964) The cross links in resilin identified as dityrosine and trityrosine, BioChem. BioPhys. Acta 93, $213-215$.

28. King, R. (2010) Dynamic Mechanical Properties of Resilin., In Engineering Science \& Mechanics, Virginia Polytechnic Institute and State University, Blacksburg.

29. Qin, G., Hu, X., Cebe, P. and Kaplan, D. L. (2012) Mechanism of Resilin Elasticity, Nature Communication 3.

30. Qin, G., Lapidot, S., Numata, K., Hu, X., Meirovitch, S., Dekel, M., Podoler, I., Shoseyov, O., and Kaplan, D. L. (2009) Expression, Cross-Linking, and Characterization of Recombinant Chitin Binding Resilin, Biomacromolecules 10, 3227-3234. 
31. Pometun, M. S., Chekmenev, E. Y., and Wittebort, R. J. (2004) Quantitative observation of backbone disorder in native elastin., J. Biol. Chem. 279, 7982-7987.

32. Khandaker, M. S., Dillard, D., and Beers, E. (in prep.) Synthesis and characterization of recombinant resilin from fruit fly.

33. Baer, M., Schreiner, E., Kohlmeyer, A., Rousseau, R., and Marx, D. (2006) Inverse temperature transition of a biomimetic elastin model: reactive flux analysis of folding/unfolding and its coupling to solvent dielectric relaxation., J. Phys. Chem. $B$ 110, 3576-3587.

34. Li, B., Alonso, D. O. V., and Daggett, V. (2001) The molecular basis for the inverse temperature transition of elastin., J. Mol. Biol. 305, 581-592.

35. Charati, M. B., Ifkovits,J. L., Burdick J. A., Linhardtc, J. G., Kiick, K. L. (2009) Hydrophilic elastomeric biomaterials based on resilin-like polypeptides, Soft Matter 5, 3412-3416.

36. Kwansa, A. L., Vita R. D., and Freeman, J. W. (2014) Mechanical recruitment of N- and C-crosslinks in collagen type I, Matrix Biology 34, 161-169.

37. Bhandarkar, M., Bhatele, A., Bohm, E., Brunner, R., Buelens, F., Chipot, C., Dalke, A., Dixit, S.,, Fiorin, G., Freddolino, P., Grayson, P., Gullingsrud, J., Gursoy, A., Hardy, D., Harrison, C.,, Hénin, J., Humphrey, W., Hurwitz, D., Krawetz, N., Kumar, S., Kunzman, D., Lee, C.,, McGreevy, R., Mei, C., Nelson, M., Phillips, J., Sarood, O., Shinozaki, A., Tanner, D.,, and Zheng, G., Zhu, F. (2011) NAMD User's Guide, Version 2.8.

38. Isralewitz, B., Baudry, J., Gullingsrud, J., Kosztin, D., Schulten, K. (2001a) Steered molecular dynamics investigations of protein function., Journal of Molecular Graphics and Modelling 19, 13-25.

39. Isralewitz, B., Gao, M., Schulten, K. (2001b) Steered molecular dynamics and mechanical functions of proteins., Current Opinion in Structural Biology 11, 224-230.

40. Gautieri, A., Buehler, M. J., and Redaelli, A. (2009) Deformation rate controls elasticity and unfolding pathway of single tropocollagen molecules., J. Mech. Behav. Biomed 2, 130-137.

41. Gautieri, A., Buehler, M.J., Redaelli, A. (2009) Deformation rate controls elasticity and unfolding pathway of single tropocollagen molecules., Journal of the Mechanical Behavior of Biomedical Materials 2, 130-137.

42. Kal'e, L., Skeel, R., Bhandarkar, M., Brunner, R., Gursoy, A., Krawetz, N., Phillips, J., Shinozaki, A., and Varadarajan, K. (1999) NAMD2: Greater scalability for parallel molecular dynamics, Journal of Computational Physics 151, 283-312.

43. Mackerell, A. D., Bashford, D., Bellott, Dunbrack, R. L., Evanseck, J. D., Field, M. J., Fischer, S., Gao, J., Guo, H., Ha, S., Joseph-Mccarthy, D., Kuchnir, L., Kuczera, K., Lau, F. T. K., Mattos, C., Michnick, S., Ngo, T., Nguyen, D. T., Prodhom, B., Reiher, W. E., Roux, B., Schlenkrich, M., Smith, J. C., Stote, R., Straub, J., Watanabe, M., Wiorkiewicz-Kuczera, J., Yin, D., and Karplus, M. (1998) All-atom empirical potential for molecular modeling and dynamics studies of proteins, The Journal of Physical Chemistry B 102, 3586-3616.

44. Jorgensen, W. L., Chandrasekhar, J., Madura, J. D., Impey, R. W., and Klein, M. L. (1983) Comparison of simple potential functions for simulating liquid water, The Journal of Chemical Physics 79, 926-935.

45. Lyons, R. E., Nairn, K. M., Huson, M. G., Kim, M., Dumsday, G., and Elvin, C. M. (2009) Comparisons of recombinant resilinlike proteins: Repetitive domains are sufficient to confer resilin-like properties., Biomacromolecules 10, 3009-3014.

46. Hess, B., Kutzner, C., Van Der Spoel, D., and Lindahl, E. (2008) GROMACS 4: Algorithms for Highly Efficient, Load-Balanced, and Scalable Molecular Simulation, J. Chem. Theory Comput. 4, 435-447.

47. Van Der Spoel, D., Lindahl, E., Hess, B., Groenhof, G., Mark, A. E., and Berendsen, H. J. (2005) GROMACS: fast, flexible, and free, J. Comput. Chem. 26, 1701-1718.

48. Kaminski, G., Friesner, R., J., T.-R., and Jorgensen, W. (2001) Evaluation and Reparametrization of the OPLS-AA Force Field for Proteins via Comparison with Accurate Quantum Chemical Calculations on Peptides, J. Phys. Chem. 105,6474 - 6487.

49. Inc, A. S. (2011) Discovery Studio Modeling Environment, 3.5 ed., Accelrys Software Inc., San Diago.

50. Humphrey, W., Dalke, A., and Schulten, K. (1996) VMD: visual molecular dynamics, Jounal of molecular graph 14, 33-38.

51. Ryckaert, J. P., Ciccotti, G., and Berendsen, H. J. (1977) Numerical integration of the cartesian equations of motion of a system with constraints: molecular dynamics of n-alkanes, Journal of Computational Physics 23, 327-341.

52. Darden, T., York, D., and Pedersen, L. (1993) Particle mesh Ewald: An N?log(N) method for Ewald sums in large systems., J. Chem. Phys. 98, 10089-10092.

53. Wolf, A., and Kirschner, K. N. (2013) Principal component and clustering analysis on molecular dynamics data of the ribosomal L11.23S subdomain, Jounal of molecular modeling 19, 539-549.

54. Pettersen, E. F., Goddard, T. D., Huang, C. C., Couch, G. S., Greenblatt, D. M., Meng, E. C., and Ferrin, T. E. (2004) UCSF Chimera--a visualization system for exploratory research and analysis., J Comput Chem. 13, 1605-1612.

55. King, S. M., and Johnson, W. C. (1999) Assigning secondary structure from protein coordinate data., Proteins: Structure, Function, and Genetics 35, 313-310.

56. Kabsch, W., and Sander, C. (1983) Dictionary of protein secondary structure: pattern recognition of hydrogen-bonded and geometrical features, Biopolymers 22, 2577-2637.

57. Gräter, F., Heider, P., Zangi, R., and Berne, B. J. (2008) Dissecting entropic coiling and poor solvent effects in protein collapse., J. Am. Chem. Soc. 130, 11578-11579. 
58. Teule, F., Furin, W. A., Cooper, A. R., R., D. J., and Lewis, R. V. (2007) Modifications of spider silk sequences in an attempt to control the mechanical properties of the synthetic fibers, Journal of Material Science 42, 8974-8985.

59. Ramachandran, G. N., Ramakrishnan, C., and Sasisekharan, V. (1963) Stereochemistry of Polypeptide Chain Configurations, J Mol Biol. 7, 95-99.

60. Pal, D., and Chakrabarti, P. (2002) On residues in the disallowed region of the Ramachandran map, Biopolymers 63, 195206.

61. Vega, M. C., Martinez, J. C., and Serrano, L. (2000) Thermodynamic and structural characterization of Asn and Ala residues in the disallowed II' region of the Ramachandran plot, PRS 9, 2322-2328.

62. Ho, B. K., and Brasseur, R. (2005) The Ramachandran plots of glycine and pre-proline, BMC Structural Biology 5, 14-24.

63. Richardson, J. S. (1981) The anatomy and taxonomy of protein structure, Adv. Ptotein Chem. 34, 167-339.

64. Dicko, C., Porter, D., Bond, J., Kenney, J. M., and Vollrath, F. (2008) Structural disorder in silk proteins reveals the emergence of elastomericity., Biomacromolecules 9, 216-221.

65. Johnson, J. C., and Korley, L. T. J. (2012) Enhanced mechanical pathways through nature's building blocks: amino acids, Soft Matter 8, 11431-11442. 


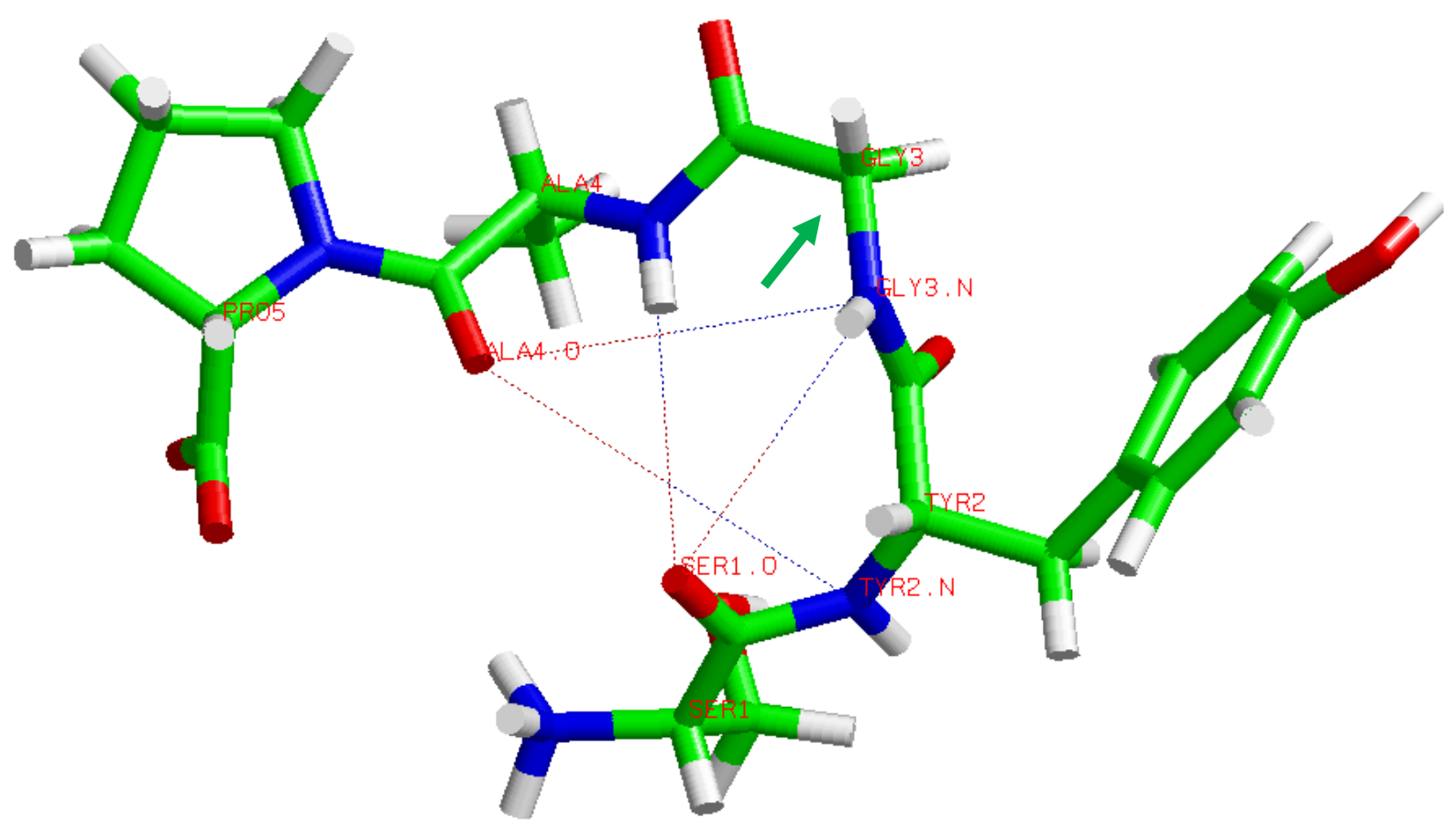

Fig. Hydrogen bonds in the repeat motif SYGAP during bent conformation. Other than proline, all the other amino acids participate in hydrogen bonding to form the stable bent structure in addition to lack of streric hindrance at glycine (arrow). 